\title{
Bücherschau.
}

Physikalisches Praktikum, von E. Wirdemann \& H. Eвеrt. 3. Auflage Braunschweig 1897. $9 \mathrm{Mk}$.

Von dem bekannten Werke ist die 3. Auflage erschienen, was gewifs zu Gunsten desselben Zeugnis ablegt. Die Verfasser sind bestrebt, wesentlich dem Bedürfnisse des Anfängers sowie derjenigen, die, wie die Chemiker überhaupt in die Lage kommen sich mit den Grundthatsachen der Physik zu beschäftigen, zu dienen. Die physikaliseh-chemischen Methoden sind hierbei thunlichst berücksichtigt. Am Schlufs des Buches sind jetzt neben den Tabellen und den Anweisungen für das logarithmische Rechnen eine Reihe von mathematischen Formeln zusammengestellt, die die Brauchbarkeit des Buches erhöhen. Es liegt in der Natur der Sache, dafs insbesondere der "elektrische Teil" wesentlich erweitert wurde. Es sind jetzt auch die Methoden zur Bestimmung der dielektrischen Eigenschaften aufgenommen. Insbesondere sei erwähnt, dafs auch der Apparat von NERNsT zur Bestimmung der Dielektrizitätskonstante Behandlung findet. In gleicher Weise sollte der Fortschritt aber auch auf anderen Gebieten der physikalischen Chemie berücksichtigt werden, und es ist daher zu rïgen, dafs die Atomgewichte auf $\mathrm{H}=1$ statt auf $\mathrm{O}=16$, bezogen sind, wic dies gegenwärtig üblich ist.

Rich. Lorent.

\section{Theorie und Praxis der analytischen Elektrolyse, von B. Neumann. Halle a. S. 1897. $7 \mathrm{Mk}$.}

Es ist ungemein wertvoll, ein Buch über analytische Elektrolyse von einem Verfasser zu besitzen, der die hierfür in Betracht kommenden Methoden kritisch und vom Standpunkte ihrer wirklichen Brauchbarkeit geprüft hat. Das vorliegende Buch ist das dankenswerte Erzeugnis einer solchen Arbeit und Kenntnis, und wird sich aus diesen Gründen bald den ihm gebührenden Eingang verschaffen. Um so mehr möchte ich aber mit meiner Ansicht, betreffend die Anfangskapitel derartiger Werke im allgemeinen und auch des vorliegenden im besondern nicht zurückhalten. Der Verfasser hätte gleich ordentlich in medias res gehen sollen. Hingegen beschäftigt er sich bis S. 95 mit allgemeinen Einleitungen, während der eigentlich sachliche (auf das Thema bezügliche) Teil des Buches bis S. 216 (Ende) geht, also nicht viel mehr, nämlich 121 Seiten, umfafst. Es wird in den ersten Teilen eine ausfübrliche "Theorie der Elektrolyse“" gegeben, die ein förmliches elektrochemisches Kolleg 
darstellt, wie schon aus den Kapitelüberschriften hervorgeht (Erscheinungen der Elektrolyse, Gesetz von Faradax, Konstitution der Elektrolyte, Wanderung der Ionen, Leitfähigkeit der Elektrolyte, Dissoziationstheorie, Vorgang bei der Elektrolyse). Dies ist meiner Ansicht nach ganz und gar unnötig. Niemand sucht diesen Stoff in einem Buch über quantitative Elektrolyse, andererseits wird niemand mit dem, was sich in solcher Kürze sagen lälst, ernstlich auftauchende Schwierigkeiten bei der quantitativen Elektrolyse bewältigen können. Solche Einleitungen holen furchtbar weit aus und bieten schliefslich sowohl zu viel, wie zu wenig. Ähnlich steht es nach meiner Meinung mit dem zweiten Abschnitt: Erzeugung, Messung und Regulierung des Stromes. Der moderne Elektroanalytiker sollte eine allgemeine Bekanntschaft mit all diesen Dingen bei seiner Wissenschaft voraussetzen und sollte sich hier darauf beschränken, das ausschliefslich notwendige anzuführen. Es könnte sonach der erste Abschnitt des Buches ganz wegbleiben, der zweite mindestens auf die Hälfte gekürzt werden. Um so prägnanter würden dann die weiteren Abschnitte hervortreten, welche den eigentlichen Gegenstand bilden und ihn, wie schon erwähnt, in ausgezeichneter Weise behandeln. Auf diese Art würde das Buch über ein Drittel dünner werden können, was ihm sehr zum Vorteil gereichen würde.

Rich. Loren:.

Tafeln zur qualitativen chemischen Analyse, von W. Hampe. Clausthal 1897.

Die bekannten und trefflichen Tafeln liegen jetzt in vierter verbesserter und vermehrter Auflage vor. Verfasser und Verleger haben keine Mühe gescheut, dieselben auf der Höhe des Fortsehrittes zu erhalten. Rich. Loren\%.

Anorganische Chemie, von F. KrafFr. Leipzig und Wien 1898. 3. Auflage. 9 Mark.

In raseher Folge hat das vorliegende Buch nun schon die dritte Auflage erlebt! Auch diesmal sei das Werk auf das angelegentlichste empfohlen.

Rich. Lorenz.

\section{Tafeln und Tabellen zur Darstellung der Ergebnisse spektroskopischer und spektrophotometrischer Beobachtungen, von TH. W. ENGEL-} MaNv. Leipzig 1897. 1 Mark 80 Pf.

Die vorliegenden, in prachtvollem Farbendruck ausgeführten Spektraltafeln sind eine ungemein willkommene Gabe für das Laboratorium. Tafel I enthält eine Reihe vorgedruckter prismatischer Spektren weilsen Lichtes, als Grundlage für die bildliche Widergabe von Absorptionsspektren. Durch Schattierung der Grundlage ist die Verteilung der Helligkeit der im Absorptionsspektrum wahrzunehmenden, möglichst gleich zu machen. Tafel II dient als Unterlage für die Darstellung des Absorptionsspektrums. Unter dem vorgedruckten farbigen prismatischen Spektrum befindet sich ein Liniensystem, in welches man die Ergebnisse der zugehörigen photometrischen Messungen einträgt. Es stehen von beiden Tafeln je 10 Exemplare zur Verfügung, wofür der angesetzte Preis ein durchaus entsprechender ist. Insbesondere bei spektrophotometrischen Bestimmungen ist ein recht reichlicher "Verbrauch" dieser Tafeln zu wünschen.

Rich. Lorenz. 